\title{
El Canal de Cultura Contemporánea de las Universidades Públicas de Andalucía: mejoras y experiencias
}

The Contemporary Culture Channel of the Andalusian public universities: improvements and experiences

\section{Jose L. Bernier Villamor (1), Gerardo Fernández Rodríguez (1), Lidia Fernández Hidalgo (1), Maypher ROMÁN DURÁN (2)}

(1) Canal de Cultura Contemporánea de las Universidades Públicas de Andalucía, Vicerrectorado de Extensión Universitaria y Deporte, Universidad de Granada 18071, portalcacocu@gmail.com.

(2) Universidad de Ciencias Informáticas (UCl), La Habana, Cuba, maypher@uci.cu

\begin{abstract}
Resumen
El Canal de Cultura Contemporánea de las Universidades Públicas de Andalucía ( $\mathrm{CaCoCu}$ ) consiste en una plataforma web con contenido cultural, fundamentalmente grabaciones audiovisuales íntegras de diversas actividades. CaCoCu se puso en explotación en 2007 y actualmente cuenta con un importante fondo documental. En este trabajo se describe el estado actual, las innovaciones tecnológicas que se han incorporado recientemente y su adaptación para nuevos estándares y una mejor representación del conocimiento, así como la metodología utilizada para la difusión del mismo mediante redes sociales.
\end{abstract}

Palabras clave: Cultura. Arte. Vídeo. Multimedia. Fondos documentales.

\section{Introducción}

El Canal de Cultura Contemporánea ( $\mathrm{CaCoCu})$ es un proyecto coordinado por la Universidad de Granada que forma parte del llamado Observatorio Atalaya (Bernier 2007), una iniciativa transversal en la que colaboran los vicerrectorados de Cultura de las diez universidades públicas de Andalucía y que cuenta con la financiación de la Consejería de Economía, Innovación, Ciencia y Empleo de la Junta de Andalucía.

Este canal consiste en una plataforma web de contenidos multimedia relacionados con las manifestaciones culturales de las distintas universidades participantes. En concreto, a través del portal se puede acceder a grabaciones de vídeo, audio, materiales pictográficos, textuales y obras de Net.Art (Bernier 2007a, 2007b).

A través de $\mathrm{CaCoCu}$, las actividades culturales son mejor rentabilizadas, ya que el aforo virtual de asistentes supera la audiencia presencial de tales actividades, por lo que las distintas universidades se han volcado en el proyecto, consiguiendo así una mayor difusión y visibilidad en Internet. La mayor parte de las actividades cul-

\begin{abstract}
The Contemporary Culture Channel of Andalusian Public Universities $(\mathrm{CaCoCu})$ is a web platform with cultural contents, that basically contains audiovisual recordings of diverse cultural activities. $\mathrm{CaCoCu}$ was first presented in 2007 and has currently an important collection of documents. This paper describes its current state, the technological innovations that have recently been developed and the adaptation of the platform to adopt new vídeo standards, a better representation of knowledge, and the methodologies used for the dissemination of the channel through social networks.
\end{abstract}

Keywords: Culture. Art. Vídeo. Multimedia. Document holdings.

turales (conciertos, obras de teatro, charlas, encuentros literarios, etc) son grabadas íntegramente en cada universidad y remitidas a $\mathrm{CaCoCu}$ donde son procesadas y puestas a disposición de los usuarios del portal (Bernier 2007a). Otra característica relevante es que estos documentos son utilizados como materiales docentes en diversas instituciones de enseñanza secundaria y universitaria, nacionales e internacionales (Bernier 2009).

CaCoCu fue desarrollado a lo largo de 2006 y se puso en explotación a principios de 2007 (Bernier 2007a, 2007b, 2009), pero no fue hasta 2008 cuando empezaron a considerarse estándares para vídeo a través de web (HTML5 2008), ni existían otras tecnologías para poder gestionar fácilmente el tipo de contenidos del portal ni la experiencia necesaria para estructurar toda la información de la variedad documental con que se iba a contar. Por ello, en 2011 se realizó un importante proceso de actualización que ha supuesto no sólo la reconstrucción íntegra del portal y el reconversión digital de todo el fondo documental, sino también un diseño nuevo de las bases de datos de y las relaciones 
entre los contenidos para poder estructurar mejor la información y el conocimiento.

Este trabajo está distribuido de la siguiente manera: en el apartado 2 se expone el estado actual del portal, reflejando el fondo documental existente y la cantidad de materiales aportados por las distintas universidades. En el apartado 3 se indican las mejoras tecnológicas realizadas recientemente, mientras que en el 4 se justifican los cambios realizados en cuanto a la estructuración del conocimiento. El apartado 5 describe la metodología de difusión utilizada para dar a conocer el canal; y, por último, en el apartado 6 se indican las pretensiones y trabajo futuro.

\section{Estado actual del canal}

Las diez universidades públicas de Andalucía colaboran proporcionando los materiales culturales originales que conforman los contenidos del portal. Para ello, cada universidad recibe una financiación anual que le permite sufragar el coste de grabación de estos acontecimientos culturales, tales como obras de teatro, conciertos, charlas, conferencias, etc y remite una copia de estas grabaciones a los técnicos de $\mathrm{Ca}$ $\mathrm{CoCu}$ (Bernier 2007a, 2007b), que son coordinados desde la Universidad de Granada.

Los técnicos de $\mathrm{CaCoCu}$ realizan fundamentalmente tres tareas: a) procesar digitalmente los materiales recibidos para transformarlos a formatos compatibles con la visualización y transmisión a través de web; b) documentar dichos materiales, completando toda la información disponible y relacionándola con otros contenidos previos; y c) difundir a través de redes sociales los contenidos e interés por el portal.

CaCoCu cuenta actualmente con más de 1.700 documentos, la mayor parte de los cuales consisten en grabaciones de vídeo con una media de duración de una hora. En la Tabla I se resume el número de materiales publicados en el portal a lo largo de estos años.

\begin{tabular}{lcc}
\hline Año & Vídeos publicados & Otros materiales \\
\hline 2007 & 111 & 251 \\
\hline 2008 & 58 & 38 \\
\hline 2009 & 76 & 0 \\
\hline 2010 & 111 & 146 \\
\hline 2011 & 273 & 222 \\
\hline 2012 & 427 & 4 \\
\hline Total & 1056 & 661 \\
\hline
\end{tabular}

Tabla I. Materiales publicados desde 2007
Puede comprobarse que la contribución de las distintas universidades, así como el fondo documental ha ido en paulatino crecimiento año tras año (el primer año hubo muchas contribuciones debido a que se aportaron materiales ya existentes producidos en años anteriores).

En la Tabla II se refleja el número de materiales aportados desde el comienzo hasta 2012 por las distintas universidades andaluzas, organizados según su tipología en grabaciones de vídeo (por ejemplo, charlas, conferencias, teatro, conciertos), grabaciones de audio (producciones musicales en $\mathrm{CD}$ ), imágenes (exposiciones, catálogos de fotografías, etc) o textos (literatura, poesía, etc).

\begin{tabular}{lcccc}
\hline Universidad & Vídeo & Audio & Imagen & Texto \\
\hline Cádiz & 270 & 39 & 107 & 16 \\
\hline Granada & 300 & 45 & 0 & 16 \\
\hline Sevilla & 65 & 18 & 0 & 6 \\
\hline Córdoba & 29 & 0 & 22 & 0 \\
\hline Internacional de Andal. & 121 & 0 & 0 & 4 \\
\hline Almería & 17 & 43 & 0 & 3 \\
\hline Jaén & 23 & 0 & 305 & 11 \\
\hline Huelva & 118 & 0 & 0 & 2 \\
\hline Pablo Olavide & 26 & 0 & 0 & 1 \\
\hline Málaga & 70 & 0 & 14 & 3 \\
\hline Otro & 17 & 0 & 0 & 1 \\
\hline TOTAL & 1056 & 145 & 448 & 65 \\
\hline
\end{tabular}

Tabla II. Materiales aportados por universidad

En la categoría "Otro" se enmarcan los documentos generados por el proyecto conjunto de las 10 Universidades Andaluzas y la televisión autonómica (Programa Tesis). Actualmente el portal sólo cuenta con tres documentos de Net.Art que se incluyen en la Tabla I pero no en la Tabla II.

Puede observarse que no todas las universidades contribuyen de idéntica manera, debido fundamentalmente a su tamaño y recursos; las universidades más pequeñas disponen de menor producción cultural, por un lado, y suelen disponer de menos infraestructuras para producción audiovisual. Aún así, la Tabla II refleja el interés de todas ellas por participar en el proyecto y difundir sus actividades culturales mediante este canal. $\mathrm{CaCoCu}$, al ser un proyecto transversal, cuenta con un mayor fondo documental audiovisual cultural que cualquiera de las universidades participantes, complementando a otros fondos documentales propios de cada 
una. De hecho, en $\mathrm{CaCoCu}$ se están añadiendo incluso colecciones antiguas del fondo histórico, que se grabaron en formatos de vídeo o audio analógico y que, gracias a la financiación recibida, se están digitalizando para difundirlos a través de Internet (por ejemplo, la colección de El Intelectual y su Memoria, de la Universidad de Granada).

Actualmente hay un ritmo de una nueva publicación diaria, y a finales de 2012 el número total de materiales en el fondo documental de Ca$\mathrm{CoCu}$ ascendía a 1.717. Todos los materiales están disponibles para ser visualizados bajo demanda por los usuarios que visitan el portal, y además están documentados con profusión de detalles para permitir su mejor localización y visibilidad en Internet.

\section{Cambios tecnológicos}

$\mathrm{CaCoCu}$ fue un proyecto pionero cuando empezó a ser desarrollado y explotado. Entonces no existían aún, o no eran suficientemente maduros, los estándares web para la difusión de vídeo en Internet, ni tampoco la tecnología (por ejemplo, el ancho de banda habitual) estaba aún lo suficientemente extendida para soportar altas resoluciones tales como la alta definición o adaptarse a distintos tipos de dispositivos visualizadores como tabletas o smartphones.

Por ello, a lo largo de 2011 se llevaron a cabo importantes cambios en cuanto a la tecnología del portal y al formato de vídeo utilizado hasta entonces con objeto de integrar los nuevos estándares y requerimientos.

\subsection{Nuevos formatos de streaming}

Inicialmente se utilizó un servidor de streaming basado en Windows Media Vídeo (Windows Media Services) que ofrecía un conjunto de ventajas frente a otras posibilidades existentes. Así, por ejemplo, usando Windows Media Server era posible transmitir vídeos de larga duración, pudiendo saltar arbitrariamente de un punto a otro de reproducción, en modo broadcasting, codificados en multistream, etc. (Bernier et al., 2007a).

Por tanto, todos los vídeos estaban codificados en formato WMV multi-stream, para poder adaptarse automáticamente al ancho de banda óptimo usado por cada visitante. El principal problema era que para poder visualizar los vídeos, los usuarios necesitaban tener instalado un complemento en su navegador, lo que en el caso de sistemas operativos basados en GNU/Linux podía resultar engorroso.
Con la aparición de estándares para transmisión de vídeo en Internet (HTML5, WebM), así como a una mayor madurez tecnológica al respecto, y pensando en la compatibilidad, se decidió cambiar el sistema de codificación y actualizar los formatos utilizados, usando un nuevo servidor de streaming y añadiendo un servidor de almacenamiento, dado el ingente volumen que ocupaba ya el fondo documental.

Tras realizar un análisis de los distintos estándares y tecnologías, se decidió modificar, por un lado, la resolución empleada en los vídeos, así como los formatos de codificación, decidiéndose codificar cada vídeo en tres formatos distintos: Sorenson Spark, H.264 y Google vídeo (WebM). En cualquiera de ellos, los vídeos se codifican con una resolución de alta definición.

El motivo para usar estos formatos es la estandarización y compatibilidad. El códec Sorenson Spark (sorenson Media) permite la reproducción de vídeo en navegadores antiguos que no incorporan el estándar HTML5. La elección de H.264 se debe a dos motivos: a) es el estándar predominante en el sector; $y, b)$ ofrece ventajas en cuanto a compresión y mecanismos de reconstrucción cuando existen pérdidas de información. Finalmente la elección de WebM se fundamenta en que, disponiendo de prestaciones similares a H.264, es el códec que usa de forma nativa el navegador de Google.

Los dos últimos formatos, H.264 y WebM, se han incluido en el estándar de vídeo de HTML5, con lo que los navegadores actuales pueden mostrarlos de forma nativa, sin necesidad de tener que instalar software adicional, como en cambio sucede con el formato Sorenson Spark.

De esta manera, para adaptar el fondo documental a estos nuevos formatos de vídeo, fue necesario recodificar de nuevo el fondo audiovisual publicado previamente. En total, fueron recodificadas más de 286 horas de vídeo en los formatos referidos. Esta ingente tarea se llevó a cabo sobre un grid de computadores (Foster y Kesselman 2003), pudiendo así realizar esta transformación en un tiempo considerablemente menor (la codificación de 1 segundo en H.264 supone 2 segundos de procesamiento en un equipo de altas prestaciones), al aprovechar los ciclos inactivos de CPU de diversos equipos que participaron en el proceso simultáneamente.

\subsection{Nuevo gestor de contenidos}

En un inicio, el portal web de CaCoCu se basaba en un gestor de contenidos muy simple, que respetaba los estándares de navegabilidad y accesibilidad, pero que se había desarrollado 
ad-hoc para poder modificar algunos modelos de página o tipos de contenidos demasiado herméticos.

Se decidió reconstruir el portal sobre un gestor de contenidos más flexible, usando finalmente UniWeb CMS (Calvo 2008, Morales 2008). Este CMS proporciona diversas ventajas frente a otros, ya que además de garantizar el cumplimiento de los estándares web y de las normativas de accesibilidad, proporciona alta disponibilidad y redundancia frente a fallos.

\subsection{Canales de usuario}

Una importante mejora que se llevó a cabo fue la posibilidad de crear canales de usuario, del mismo modo a como lo hace YouTube (Canales Youtube). De esta forma, es factible que cada universidad disponga de su canal propio en $\mathrm{CaCoCu}$, mostrando preferentemente los materiales de dicha universidad. Así, las distintas universidades pueden utilizar accesos personalizados a $\mathrm{CaCoCu}$, donde todos los contenidos están particularizados para ellas (por ejemplo, las novedades, los más populares, las búsquedas y listados, etc.).

Los usuarios registrados también pueden crear sus propios canales, seleccionando sus vídeos preferidos para que sean accedidos directamente por otros usuarios.

Por otra parte, los videos permiten ser incrustados en otras plataformas web, lo cual es utilizado por las distintas universidades que, de esta manera, pueden mostrar los videos de $\mathrm{CaCoCu}$ en sus propias plataformas, sin tener que disponer de una copia en sus servidores.

\subsection{La "Cacocupedia"}

Tal y como se ha mencionado previamente, CaCoCu no es sólo un canal de TV cultural bajo demanda, sino también un fondo documental donde quedan registradas las actividades culturales organizadas por las distintas universidades, y en el cual, además del contenido audiovisual, se almacenan descripciones muy completas de estas actividades (Bernier et al., 2009).

Relacionar los contenidos masiva y redundantemente mediante hipertexto de forma similar a como hace la Wikipedia era un mecanismo intuitivo para facilitar la localización de contenidos y mejorar la experiencia de usuario.

Así pues, además de las fichas descriptivas de cada documento, se crean adicionalmente fichas descriptivas de autores, entidades, salas, universidades, etc. Por ejemplo, al consultar un documento y seleccionar al autor mediante un enlace, la ficha de dicho autor muestra información sobre el mismo y enlaces a otros documentos publicados en $\mathrm{CaCoCu}$, que a su vez se relacionan mediante enlaces con las distintas universidades responsables del evento, etc.

De esta forma, mediante la Cacocupedia, el canal ofrece una forma alternativa de localizar los contenidos, más intuitiva y natural.

\section{Representación interna de los contenidos}

Un aspecto importante en $\mathrm{CaCoCu}$ es la forma de representar la información y relacionar los contenidos entre sí. La variedad de contenidos en $\mathrm{CaCoCu}$ es grande y, sobretodo, es heterogénea la forma de describir los mismos, ya que las actividades culturales que abarca son muy diversas.

Se analizaron distintas posibilidades para la documentación de los materiales de una forma estandarizada: Dublin Core (Dublin Core Metadata Initiative), MARC (MARC standards) y Encoded Archival Description (EAD), y se decidió implementar una solución mixta que integrase toda la variedad de documentos descrita con anterioridad.

Así, para representar cada documento, se distinguen dos partes fundamentales: agregador y elemento. El agregador es común a varios elementos, pudiendo incluso contener información heredada de otros agregadores. El agregador almacena la información siguiente: título, descripción, imagen previa, universidad organizadora, colaboradores, organizadores, lugar, fecha, categoría, agregador padre, tipo (ciclo, colección y documento), y subtipo (vídeo, audio, imagen, texto y netart).

El motivo para usar esta jerarquía es que a menudo coexisten actividades de diversa índole (tanto en tiempo, como en tipología de materiales 0 actividades, etc.) sobre una temática común. Por ejemplo, hay un evento denominado Congreso Internacional Doceañista consistente en un ciclo de conferencias sobre la Constitución de 1812, que se complementa con un conjunto de exposiciones relacionadas. Además, esta actividad se repite cada año y en cada edición se presentan algunas particularidades.

Con el uso del concepto de agregador se pretende suprimir la redundancia de información, ya que la información de cada elemento se complementa mediante el agregador al que pertenece, además de permitir relacionar distintos elementos entre sí. 
Es importante señalar que el sistema permite varios formatos de fecha, ya que un problema que se presentó es que hay actividades antiguas, de las que no existe apenas documentación actualmente y no era posible precisar exactamente una fecha concreta. Por ello, se diseñó la posibilidad de indicar una fecha puntual, un rango de fechas o una descripción genérica aproximada.

Por su parte, cada elemento contiene información adicional a la anteriormente mencionada. Según el tipo de documento, esta información varía. La Tabla III resume los campos adicionales considerados para cada tipo de documento y que completan la estructura documental.

\begin{tabular}{ll}
\hline Tipo & Campos adicionales \\
\hline Vídeo & $\begin{array}{l}\text { Duración, responsable grabación, idiomas (audio } \\
\text { y subtítulos), url_vídeo, ancho, alto }\end{array}$ \\
\hline Audio & Duración, url_audio \\
\hline Imagen & $\begin{array}{l}\text { Soporte, técnica, ancho, alto, profundidad, } \\
\text { unidad, url_imagen }\end{array}$ \\
\hline Texto & Isbn, issn, páginas, editorial, url_documento \\
\hline Net.Art & $\begin{array}{l}\text { Técnica, url_imagen_previsualización, } \\
\text { url_documento }\end{array}$ \\
\hline
\end{tabular}

Tabla III. Información específica según tipo de documento

Otros conceptos, tales como los autores de las obras, también se definen mediante diversos campos informativos: nombre, apellidos, pseudónimo, año de nacimiento, lugar de nacimiento, nacionalidad, profesión, biografía, imagen.

De manera adicional, cada elemento dispone de información sobre los gustos de los usuarios como descriptores, número de visitas, comentarios y ranking.

\section{Difusión de $\mathrm{CaCoCu}$}

Una importante tarea para promocionar el portal se relaciona con su difusión. En este sentido, aparte de campañas de difusión convencionales, tales como la presencia en ferias estudiantiles, la cartelería o el envío masivo de correo electrónico en cada universidad, el mayor esfuerzo de difusión se realiza a través de redes sociales y campañas en medios sociales.

Se explotan básicamente tres estrategias: mantenimiento de un blog; perfiles en Facebook, Twitter y Google+; y campañas en medios sociales

\subsection{El blog de $\mathrm{CaCoCu}$}

El blog se usa como complemento informativo al portal, con entradas relacionadas con temas transversales a los contenidos puramente documentales.

Las entradas del blog describen en mayor profundidad y de forma más distendida determinados eventos culturales, o contienen pequeñas entrevistas a responsables o autores de la actividad, o bien se dedican a otras informaciones relacionadas (por ejemplo, la presencia de Ca$\mathrm{CoCu}$ en una feria, la visita a una universidad, el inicio de una campaña en medios sociales, etc.).

El blog permite llegar a los usuarios de una forma más personal, además de proporcionar una mayor visibilidad en Internet de los contenidos del portal, ya que son referenciados desde las entradas correspondientes.

\subsection{Perfiles en Facebook y Twitter}

Facebook y Twitter se han revelado como unas importantes herramientas para la difusión y promoción (Goh, Heng and Lin 2013). En CaCoCu se usan perfiles en estas dos redes sociales, y aunque la filosofía de uso es distinta en ambas, el objetivo común es llegar a más usuarios y conseguir fidelizarlos.

Facebook se usa como una red generalista, donde a diario se publicitan las novedades del portal, por ejemplo, los últimos documentos añadidos, los próximos eventos, etc., mientras que Twitter se usa como un referenciador.

La Tabla IV resume la actividad desarrollada para difusión en las distintas redes sociales.

\begin{tabular}{lccc}
\hline Medio & $\begin{array}{c}\text { Número de } \\
\text { entradas }\end{array}$ & $\begin{array}{c}\text { Frecuencia de } \\
\text { actualización }\end{array}$ & Filiaciones \\
\hline Blog & 155 & $1 /$ semana & \\
\hline $\mathrm{Fb}$ & & $6 /$ semana & 748 "Me gusta" \\
\hline Twitter & 3.920 & $15-20 /$ día & 314 seguidores \\
\hline $\mathrm{g}+$ & 174 & $4-8 /$ día & 30 "+1" \\
\hline
\end{tabular}

Tabla IV. Resumen actividad en redes sociales

\subsection{Campañas en Medios Sociales}

Con objeto de promocionar $\mathrm{CaCoCu}$, en un inicio se desplegaron campañas de publicidad contextual usando Google Adwords.

Se ha comprobado una mayor efectividad para fidelizar usuarios diseñando campañas basadas en redes sociales. En estas campañas, a cambio de que el usuario seleccione un "Me gusta" o un " +1 " se puede participar en un concurso para 
ganar algún premio (por ejemplo, un bono de entradas para el cineclub universitario). Este tipo de campañas no son económicamente costosas y consiguen una alta penetración horizontal en las distintas universidades participantes, ya que son los propios usuarios quienes realizan la difusión a través de sus propios perfiles en las redes sociales de forma viral.

\section{Conclusiones y trabajo futuro}

$\mathrm{CaCoCu}$ es un proyecto transversal a todas las universidades públicas andaluzas, que permite mantener un registro documental de las actividades culturales organizadas y difundir grabaciones audiovisuales de las mismas a través de Internet. El impacto que tiene $\mathrm{CaCoCu}$ es superior al que tienen en la mayoría de los casos estas actividades de forma presencial, aumentando de esta forma el público que puede participar en ellas y permitiendo que estos materiales puedan ser utilizados como complemento educativo.

Como nuevos objetivos para mejorar el canal, se han planteado los siguientes proyectos:

7. Desarrollo de un sistema que automáticamente exporte a YouTube un extracto de cada documento audiovisual publicado en CaCoCu junto a su descripción.

8. Adaptación de las descripciones a formatos documentales estándares para permitir el intercambio de conocimientos con otras plataformas.

9. Desarrollo de un sistema de recomendación que detecte los gustos o afinidades de los usuarios y pueda facilitarles la localización de materiales de su interés.

10.Estudio experto de la usabilidad y experiencia de usuario del portal con objeto de mejorar la distribución de contenidos y optimizar la navegación y localización de recursos.

\section{Referencias}

Bernier, Jose Luis; Gómez-Domínguez, David; FernándezRodríguez, Gerardo; et al. (2007a). CaCoCu: un portal web para la difusión de contenidos multimedia culturales de las universidades públicas andaluzas. // El Profesional de la Información. 16:3 (Mayo-Junio 2007) 216-224.

Bernier, Jose Luis; Gómez -Domínguez, David; FernándezRodríguez, Gerardo (2007b). El portal web CaCoCu: un canal de cultura contemporánea de las universidades públicas andaluzas // Ibersid (2007) 151-156.

Bernier, Jose Luis; García-Mendoza, Sara; Barcheín, Mario; Rojas, Ignacio (2009). CaCoCu: a multimedia channel to disseminate the contemporary culture as a source of educational materials. // Research, Reflections and Innovations in Integrating ICT in Education. 1 (2009) 16-20.

Calvo, Jose Carlos; Barcheín, Mario; Morales, Antonio; García, Iván; Bernier, Jose Luis. Platform for building and managing large websites with distributed information. // Research, Reflections and Innovations in Integrating ICT in Education. 2 (2009) 1011-1015.

Canales Youtube. http://www.youtube.com/yt/advertise/ es/channels.html (2013-04-02)

Dublin Core Metadata Initiative. http://dublincore.org/ (201304-02)

EAD Encoded Archival Description. Washington: The Library of Congress. http://www.loc.gov/ead/ (2013-04-02)

Foster, lan; Kesselman, Carl (2003). The Grid 2, Second Edition: BluePrint for a New Computing Infraestructure //Elsevier Series in Grid Computing (2003).

Goh, K. Y.; Heng, C. S.; Lin, Z. J. (2013). Social Media Brand Community and Consumer Behavior: Quantifying the Relative Impact of User- and Marketer-Generated Content. // Information Systems Research. 24:1 (2013) 88-107.

HTML5. W3C Working Draft 22 January 2008. http://www.w3.org/TR/2008/WD-html5-20080122 (201304-02).

MARC Standards. Washington: The Library of Congress. http://www.loc.gov/marc/ (2013-04-02).

Morales, Antonio; Calvo, Jose Carlos; García, Iván; Barcheín, Mario; Bernier, Jose Luis (2009). An institutional web 2.0 platform for academic purposes: case study. // Research, Reflections and Innovations in Integrating ICT in Education. 2 (2009) 912-916.

Sorenson Media. Sparc SDK. http://www.sorensonmedia. com/vídeo-codec (2013-04-02)

WebM: an open web media project. http://www. webmproject.org/ (2013-04-02)

Windows Media Services 9 Series. http://www.microsoft .com/windows/windowsmedia/forpros/server/server.aspx (2013-04-02)

Enviado: 2012-04-05. Segunda versión: 2013-06-28. Aceptado: 2013-08-23. 